\title{
PLEIOTROPIC ROLE OF SIMVASTATIN AND ALENDRONATE ON MESENCHYMAL STEM CELLS
}

\author{
RIHAM M ALY ${ }^{1,2 *}$, MARWA M ELLITHY ${ }^{1}$, DINA SABRY ${ }^{3}$ \\ ${ }^{1}$ Department of Basic Dental Science, Oral and Dental Research Division, National Research Centre, Cairo, Egypt. ${ }^{2}$ Stem Cell Laboratories, \\ Centre of Excellence for Advanced Sciences, National Research Centre, Cairo, Egypt. ${ }^{3}$ Department of Medical Biochemistry and \\ Molecular Biology, Faculty of Medicine, Cairo University, Cairo, Egypt. Email: riham.aly@gmail.com
}

Received: 12 September 2018, Revised and Accepted: 25 October 2018

\section{ABSTRACT}

Objectives: Previously, our research group has investigated the effect of alendronate (ALN) and simvastatin (SV) in their minimum inhibitory dose $\left(\mathrm{IC}_{50}\right)$ on oral squamous cell carcinoma cell line where inhibition of angiogenesis has been demonstrated. In the present study, we further investigate the effect of the previously calculated $\mathrm{IC}_{50}$ of SV and ALN and their combination on two different types of stem cells to show that the same drug may have different effects on different cells.

Methods: Stem cells were isolated from rat adipose tissue and oral mucosa. After passaging, cells were subjected to Aln, Sv separately as well as combined in their half maximal inhibitory concentration $\left(\mathrm{IC}_{50}\right.$ ). 3-[4,5-dimethylthiazol-2-yl]-2,5 diphenyl tetrazolium bromide assay was performed to evaluate cytotoxicity. After seven days, osteogenic differentiation was evaluated using alizarin stain. Real time polymerase chain reaction was used to evaluate Osteopontin gene expression.

Results: Our results demonstrated that the proposed combination of Aln and Sv in their IC50 enhanced the osteogenic differentiation of both types of stem cells.

Conclusion: The combined effect of Aln and Sv may represent a novel pharmacological approach in treating bone metastasis and at the same time treating the cancer itself.

Keywords: Stem cells, Adipose tissue, Oral mucosa-simvastatin, Alendronate-osteogenesis.

(C) 2018 The Authors. Published by Innovare Academic Sciences Pvt Ltd. This is an open access article under the CC BY license (http://creativecommons. org/licenses/by/4. 0/) DOI: http://dx.doi.org/10.22159/ajpcr.2018.v11i12.29697

\section{INTRODUCTION}

Although the therapeutic utilization of stem cells is emerging expeditiously as a regenerative option to treat diverse aggressive and fatal diseases such as cancer, until now surgery has been the gold standard in treating cancerous tissue. Cancer-treating regimens and protocols often resort to chemotherapy with or without radiotherapy as an adjuvant to surgery. The sarcomas of bone, especially, present a very challenging type of tumors since tumor removal that is often indicated is usually followed by bone fracture. Moreover, some sort of resistance to chemo/radiotherapy usually develops. Alendronate (ALN), a member of bisphosphonates family, is a potent inhibitor of bone resorption that has been widely administered in conditions such as malignant hypercalcemia, osteolytic bone diseases, and osteoporosis [1]. ALN has also been reported to exert a significant and dose-dependent effect on osteoblastogenesis of human mesenchymal stem cells (MSCs) [2]. Similarly, statins are considered potential therapeutic agents for antiosteoporotic treatment. Preclinically, ALNs were demonstrated to play an effective and safe therapeutic role when used as an adjuvant agent to chemotherapy and/or radiotherapy approaches routinely used in the management of head and neck squamous cell carcinoma [3,4]. Recent in vitro studies on bone marrow stromal cells, embryonic stem cells, and osteoblast-like cells have illustrated the anabolic action of statins exert on bone through enhancing bone buildup [5]. It was discovered that treatment with statins can stimulate cellular osteogenic differentiation by enhancing Bone Morphogenic protein-2 (BMP-2) mRNA expression in addition to increasing trabecular bone volume when orally administered to ovariectomized rats. However, controversial results still exist regarding the biological effect of statins on bone metabolism which may be attributed to the different kinds of statins available on the market and different dosages and administration methods [6]. Recent studies have shown that statins are capable of inducing osteogenic differentiation through increasing cell rigidity in MSCs and that combined application with osteoconductive materials may asset stem cell-based bone tissue engineering [7]. Our group previously demonstrated that combining statins with ALNs synergistically resulted in the death of cancerous cells of squamous cell carcinoma [8]. In the present study, we set to investigate our hypothesis that the combination of those two drugs exhibits dual effect depending on the type of cells. Here, we investigate the potential of this combination to induce osteogenic differentiation when administered to MSCs from the oral mucosa and adipose tissue using real-time polymerase chain reaction (RT-PCR), which is a technique used to monitor the progress of a PCR reaction in RT while quantifying a relatively small amount of osteogenic genes. Furthermore, we assessed the enhancing effect of this combination of drugs on cell viability using the colorimetric 3-(4,5-Dimethylthiazol-2-yl)-2,5-diphenyltetrazolium bromide (MTT) assay for assessing cell metabolic activity. MTT enables simple and reliable counting of metabolically active cells while indicating their viability and proliferation rate. Its main advantages are its rapidity, accuracy, and the lack of any radioisotopes [9].

\section{MATERIALS AND METHODS}

\section{Materials and reagents}

ALN (4-amino-1-hydroxybutylidiene-1, 1-bisphosphonate) preparation: Stock solutions of the compounds ( 10 or $20 \mathrm{mM}$ ) were prepared in distilled water ( $\mathrm{pH}$ adjusted to 7.4) and filter-sterilized using a $0.2 \mu \mathrm{m}$ filter. Simvastatin (SV) preparation: Stock solutions of the compound were prepared by dissolving the powder in dimethyl sulfoxide not exceeding $0.1 \%$.

\section{Stem cell isolation and culture}

Stem cells used in this study were isolated from fat tissue excised from the inguinal pad of fat of mice as well as from their buccal oral mucosa. The minced pieces were collected in sterile, labeled, falcon 
tube. Enzymatic digestion method was used to isolate stem cells from extirpated adipose and oral tissue. Tissues were digested with collagenase II (R and D) and were kept in an incubator at $37^{\circ} \mathrm{C}$ for $90 \mathrm{~min}$ while shaking on a shaker every $15 \mathrm{~min}$. The digestion reaction was neutralized by adding complete culture medium supplemented with fetal bovine serum. Neutralized cells were centrifuged at $2300 \mathrm{rpm}$ for $5 \mathrm{~min}$. The pellet was resuspended by adding $1 \mathrm{ml}$ of routine culture medium and pipetting several times. Resuspended cells were then passed through a 70- $\mu \mathrm{m}$ cell strainer (BD Sciences) before plating (counting) to remove debris and ensure making a single cell suspension. The cells were resuspended at a density of $10,000 \mathrm{cells} / \mathrm{cm}_{2}$ in complete culture media containing $\alpha$-MEM with L-glutamine, (Gibco, in vitro gen Life Technologies, USA) supplemented with $10 \%$ fetal bovine serum (Gibco, in vitro gen Life Technologies, USA), antibiotics (penicillin G, $100 \mathrm{U} / \mathrm{ml}$, and streptomycin, $100 \mu \mathrm{g} / \mathrm{ml}$ ), and antimycotic agent (Fungizone, $0.25 \mu \mathrm{g} / \mathrm{ml}$ ) in sterile $25 \mathrm{~cm}^{2}$, polystyrene, filter cap, cell-culture plates labeled by cell type and date and incubated in a $\mathrm{CO}_{2}$ incubator at $37^{\circ} \mathrm{C}$ in a humidified atmosphere of $5 \% \mathrm{CO}_{2}$. Cells were observed for proliferation and cell morphology twice weekly using an inverted light microscope. The cell culture medium was changed as needed (every 3-4 days). Once the cells reached $70-80 \%$ confluence, they were passaged using trypsin/EDTA solution. Cell cultures from the third passage were used for differentiation assessment.

\section{Drug treatment and grouping}

Minimum inhibitory dose of SV was $6.534 \mu$ mole while that of ALN was $30.56 \mu$ mole as previously calculated by Abd-el-Fattah et al. [8]. Group 1: Adipose MSCs (AMSCs) in complete culture medium which served as control; Group 1a: AMSCs+ALN, Group 1b: AMSCs+SV, and Group 1c: AMSCs+(ALN+SV combination). Group 2: Oral MSCs which served as control; Group 2a: Oral MSCs+ALN, Group 2b: Oral MSCs+SV, and Group 2c: Oral MSCs+(ALN+SV combination).

\section{Cell viability assessment}

MTT reagent and detergent solution were obtained from TACSTM TREVIGEN@ 8405 Hegerman Ct. Gaithersburg, supplied ready for use according to the manufacturer's instructions. The MTT reagent was added $(10 \mu \mathrm{l}$ per well), and the plate was incubated for $2-4 \mathrm{~h}$ to allow for intracellular reduction of the soluble yellow MTT to the insoluble purple formazan dye. When the purple precipitate was clearly visible under the microscope, the detergent reagent was added (100 $\mu$ l per well) to solubilize the formazan dye. The quantity of formazan product as measured by the amount of $490 \mathrm{~nm}$ absorbance is directly proportional to the number of living cells in culture plates. Culture plates were left with cover in the dark for 2-4 h at room temperature. The plate cover was removed, and the absorbance in each well was measured. The optical density values were measured at a range from $490 \mathrm{~nm}$ to $630 \mathrm{~nm}$ using an enzyme-linked immunosorbent assay plate reader (Dynatech MRX 5000; Dynex, Chantilly, VA).

\section{Alizarin red staining}

Alizarin red stain $(2 \mathrm{~g})$ and distilled water $(100 \mathrm{ml})$, the two constituents were mixed well and the $\mathrm{pH}$ was adjusted to 4.1-4.3 with $10 \%$ ammonium hydroxide. On day 7 of culture, the induced cells were stained using Alizarin Red stain to identify mineralization. The medium was carefully aspirated from each plate to avoid aspirating the cells, and then, the cells were washed with PBS. The cells were then fixed by incubating on ice-cold $70 \%$ ethanol for $1 \mathrm{~h}$ at room temperature. The alcohol was carefully aspirated and rinsed twice (5-10 min each) with distilled water. The water was aspirated, and Alizarin Red solution was added to cover the plates ( $500 \mu \mathrm{l}-1 \mathrm{ml}$ per plate). The plates were incubated at room temperature for $30 \mathrm{~min}$. The Alizarin Red solution was removed and the plates were washed 4 times with $1 \mathrm{ml}$ distilled water. Finally, 1-1.5 ml distilled water was added to each plate to prevent the cells from drying. The plates were ready for visual inspection and image acquisition. The plates were monitored using inverted phase contrast microscope, and digital micrographs were taken.

\section{Osteopontin gene expression by quantitative-RT-PCR}

The total RNA was extracted from the cultured cells, and RT-PCR was performed to analyze the mRNA level of osteoblastic differentiation marker gene; Osteopontin (Forward: 5'AGATGATGGTGCAGAGGAA-3', Reverse: 5'GGTGGTTCTGGCAGGGATTT-3').

\section{Statistical analysis}

The collected data were tabulated using Microsoft Excel (Microsoft Office, 2007). Analysis of variance was used to test the significance of difference between different groups. Tukey's test was used to test the significance of difference between groups. The level of $\alpha$ was 0.05. All statistical analysis was performed using SPSS for windows ver.10.1.

\section{RESULTS}

\section{Stem cell characterization}

Following the isolation and culturing procedures, cells were kept for one week before they started to attach to the bottom of culture dishes. The cells exhibited spindle-like or stellate-shaped appearance reflecting the diverse morphology of isolated cells (Fig. 1). Cells continued to proliferate and propagate until $70-80 \%$ of the dish area became covered with cells by day 16 (confluence $70-80 \%$ ). Following confluence, the cells were passed successfully up to the third passage. Cells maintained their stellate and spindle-like appearance indicating an undifferentiated state in the conventional culture media.

\section{ALN and SV combination inhibited cell viability}

In Group1 (adipose-derived MSCs [ASCs]), there was no significant difference between cell viability of Groups $1 \mathrm{a}$ and $\mathrm{b}$ as shown by Tukey's test (Table 1 and Fig. 2). Furthermore, there was no significant difference between each group and control group. There was a significant difference between Group1c and each of the previous groups, indicating that combining ALN and SV inhibited cell viability of ASCs. Similarly, Group 2 (oral mucosa MSCs) illustrated the same results where there was no significant difference between cell viabilities of
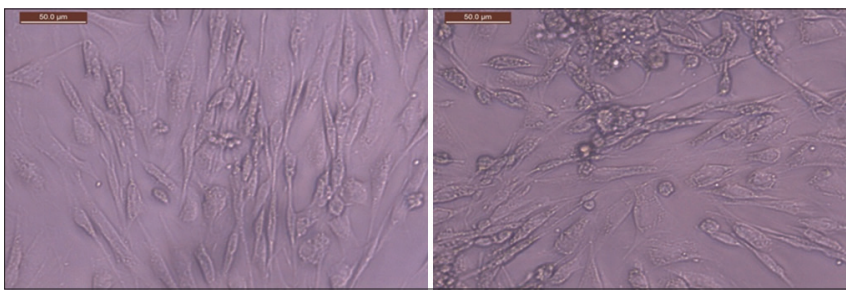

Fig. 1: Isolated oral mesenchymal stem cells (left) and adiposederived mesenchymal stem cells (right) in the third passage at 70-80\% confluence. Cells are showing typical stellate and spindleshaped appearance of mesenchymal stem cells (scale bar $50 \mu \mathrm{m}$ )

Table 1: Tukey's multiple comparisons test comparing cell viability between different sub-groups of AMSCs as assessed by MTT assay

\begin{tabular}{lllll}
\hline & Adipose Control & Aln+adipose cells & Sim+adipose & Adipose MSCs+simvastatin+Alendronate \\
\hline Mean \pm SD & $1.416 \pm 0.254$ & $1.452 \pm 0.236$ & $1.6 \pm 0.088$ & $0.7086 \pm 0.5761$ \\
F & & & \\
P & 11.04 & $0.2972^{\dagger}$ & $1.535^{\dagger}$ & $5.901^{*}$ \\
Adipose Control vs. Tukey's test (q) & & $1.238^{\dagger}$ & $6.198^{*}$ \\
Aln+adipose cells & & & $7.436^{*}$ \\
Sim+adipose & & & \\
\hline
\end{tabular}


Table 2: Tukey's multiple comparisons test comparing cell viability between different sub-groups of OMSCs as assessed by MTT assay

\begin{tabular}{lllll}
\hline & Oral Control & Aln+Oral cells & Sim+Oral cells & Oral mucosa MSCs+simvastatin+Alendronate \\
\hline Mean \pm SD & $1.252 \pm 0.231$ & $1.632 \pm 0.322$ & $1.531 \pm 0.921$ & $0.2628 \pm 0.09827$ \\
F & 12.33 & & & \\
P & $<0.0001^{*}$ & $2.134^{\dagger}$ & $1.567^{\dagger}$ & $5.554^{*}$ \\
Oral Control vs. Tukey's test (q) & & $0.5671^{\dagger}$ & $7.688 *$ \\
Aln+Oral cells & & & $7.121^{*}$ \\
Sim+Oral cells & & & \\
\hline
\end{tabular}

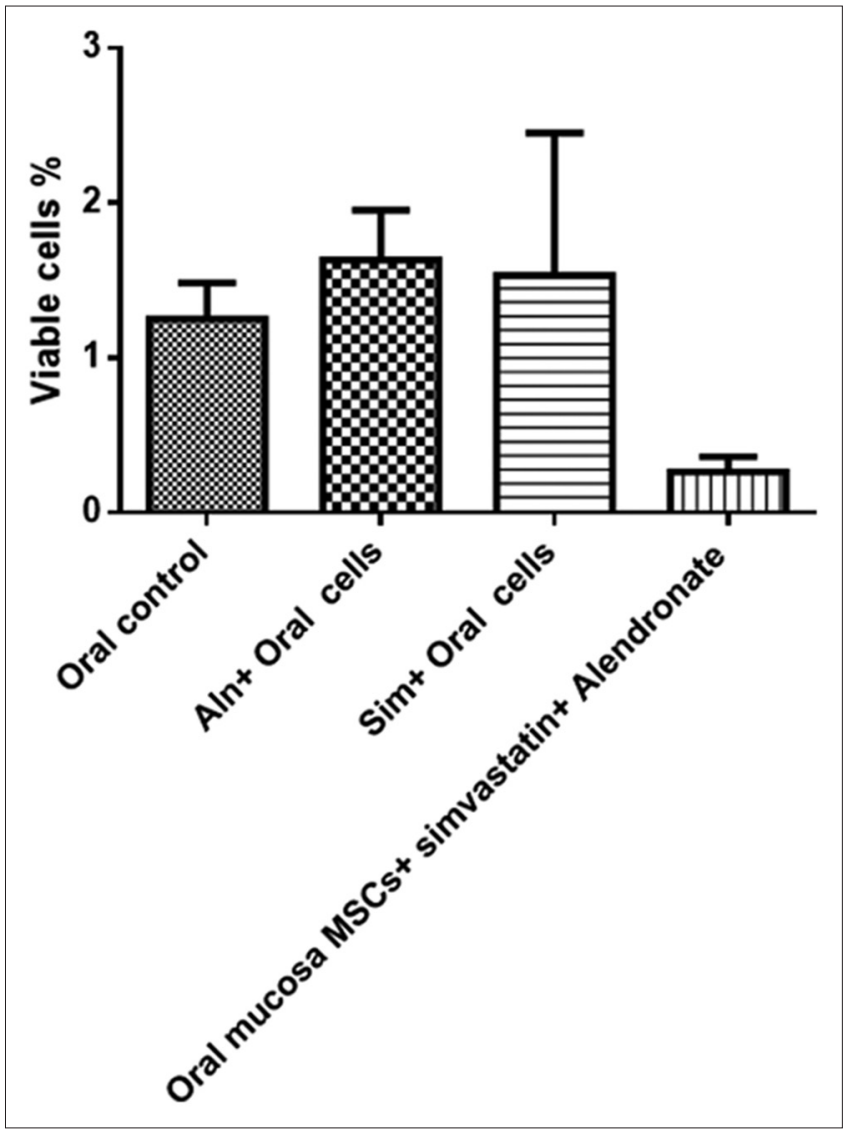

Fig. 2: Histogram illustrating cell viability in AMSCs sub-groups as assessed by MTT assay

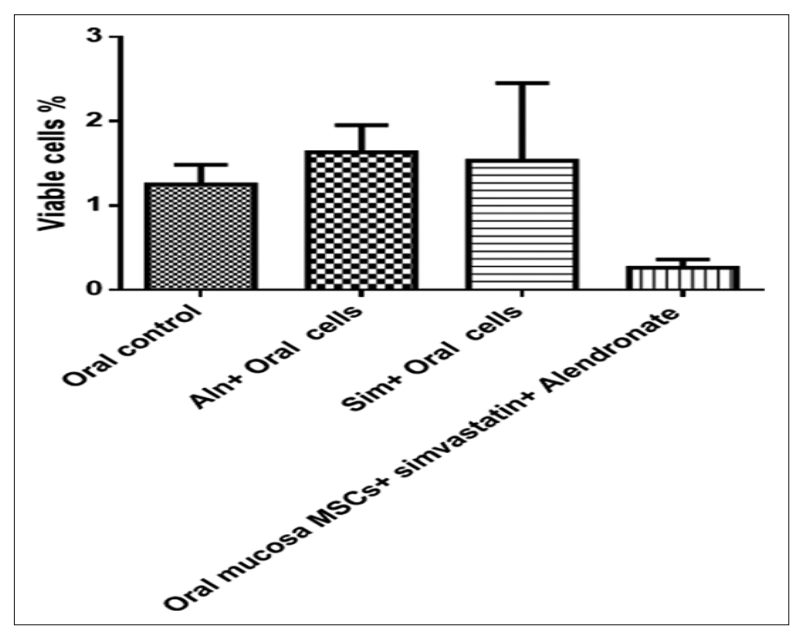

Fig. 3: Histogram showing cell viability in OMSCs sub-groups as assessed by MTT assay

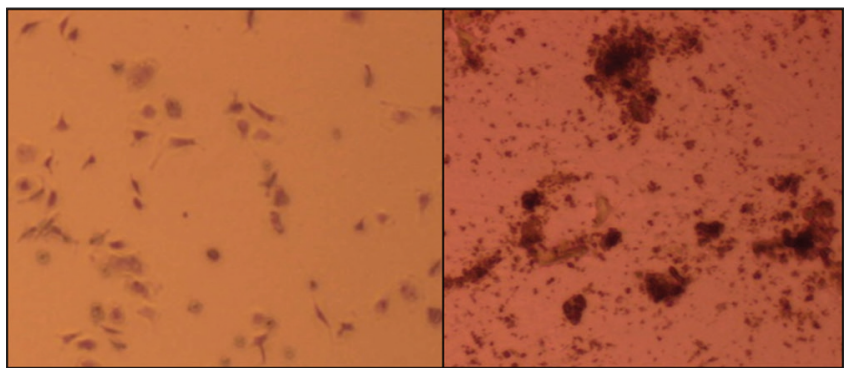

Fig. 4: Alizarin red stain of oral mesenchymal stem cells (left) and adipose derived mesennchymal stem cells (right). Positive results are indicated by intense orange red staining of calcific nodules (Scale Bar 50 $\mu \mathrm{m}$ )

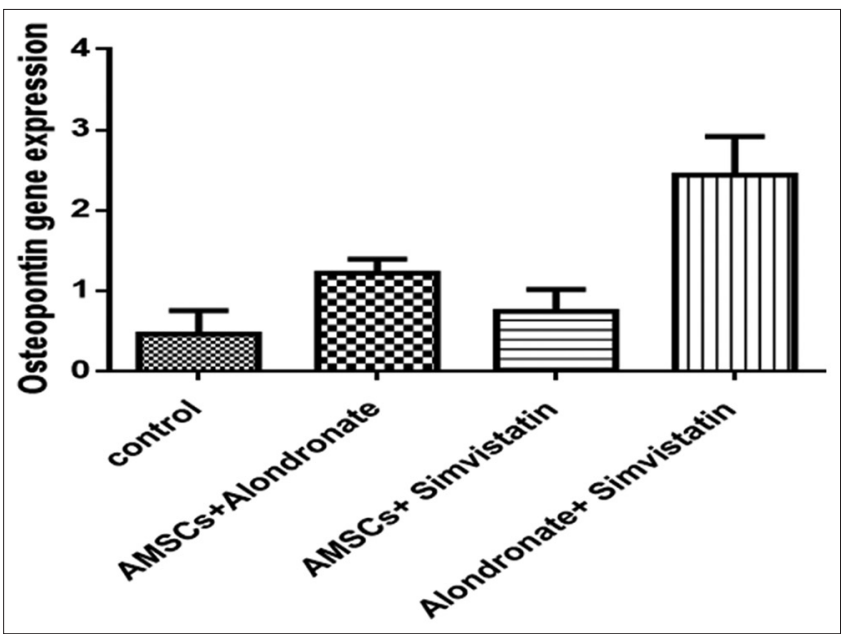

Fig. 5: Histogram illustrating Osteopontin gene expression via RtPCR in AMSCs sub-groups

(Fig. 3). Furthermore, there was no significant difference between each group and the control group. There was a significant difference between Group 2c and each of the previous groups, indicating that combining ALN and SV inhibited cell viability (Table 2).

SV and ALN combination induced osteogenic differentiation Alizarin red stain results showed minerals formation and confirmed osteogenic differentiation. Cells demonstrated orange-red colorimetric changes indicating calcium crystal deposition and successful osteogenic differentiation of isolated stem cells (Fig. 4). The osteogenic gene expression of Osteopontin revealed a highly significant difference between Groups 1c and 1a than from Groups 1 and $1 \mathrm{~b}$ as shown by Tukey's test (Table 3 and Fig. 5). Furthermore, there was a significant difference between Group 1c and each of the previous groups, indicating that combining ALN and SV highly enhanced the Osteopontin gene expression of ASCs. Similarly, there was a highly significant difference between Osteopontin gene expression of Groups 2c and 2a than from Groups 2 and $2 \mathrm{~b}$ as shown by Tukey's test. Furthermore, there was 
Table 3: Tukey's multiple comparisons test comparing Osteopontin gene expression via Rt-PCR in different sub-groups of group 1

\begin{tabular}{lllll}
\hline & Adipose Control & Aln+adipose cells & Sim+adipose & Adipose MSCs+simvastatin+Alendronate \\
\hline Mean \pm SD & $1.416 \pm 0.254$ & $1.452 \pm 0.236$ & $1.6 \pm 0.088$ & $0.7086 \pm 0.5761$ \\
F & & & \\
P & 11.04 & $0.2972^{\dagger}$ & $1.535^{\dagger}$ & $5.901^{*}$ \\
Adipose Control vs. Tukey's test (q) & & $1.238^{+}$ & $6.198^{*}$ \\
Aln+adipose cells & & & $7.436^{*}$ \\
\hline
\end{tabular}

Table 4: Tukey's multiple comparisons test comparing Osteopontin gene expression via Rt-PCR in different sub-groups of group 2

\begin{tabular}{lllll}
\hline & Control & MSCs+Alondronate & MSCs+ Simvistatin & Alondronate+ Simvistatin \\
\hline Mean \pm SD & $0.4068 \pm 0.2121$ & $1.013 \pm 0.4773$ & $0.2631 \pm 0.1369$ & $1.978 \pm 0.3242$ \\
$\mathrm{~F}$ & 73.51 & & & $17.28^{*}$ \\
$\mathrm{P}$ & $<0.0001^{*}$ & $6.668^{*}$ & $1.581^{\dagger}$ & $10.61^{*}$ \\
Control vs. Tukey's test (q) & & & $8.249^{*}$ & $18.86^{*}$ \\
MSCs+Alendronate & & & \\
MSCs+Simvistatin & & & & \\
\hline
\end{tabular}

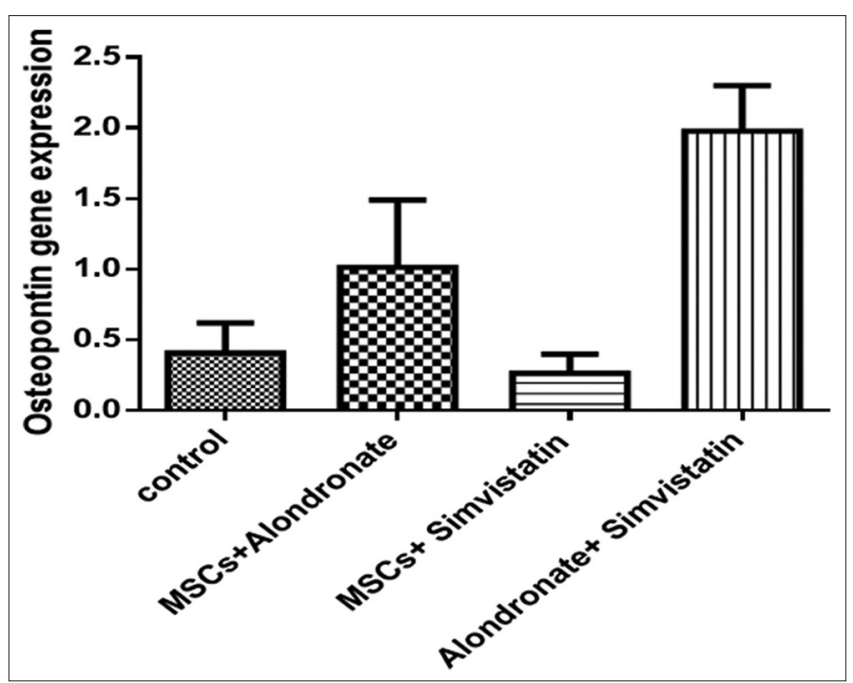

Fig. 6: Histogram showing Osteopontin gene expression in OMSCs sub-groups

a significant difference between Group 2c and each of the previous groups, suggesting that combining ALN and SV highly enhanced the Osteopontin gene expression of oral MSCs (Table 4 and Fig. 6).

\section{DISCUSSION}

In this study, we sought to investigate the potential role of a novel combination of SV and ALN in inducing osteogenic differentiation of stem cells. Routinely, the used osteogenic media constitutes dexamethasone, a corticosteroid derivative that can elicit negative effects on healing potential if applied clinically [10]. Previous studies and researches have concluded the effect of bisphosphonates on treatment of bone resorption. This effect was mediated through the inhibition of osteoclastic function [11]. Furthermore, the family of Statins has shown an anabolic impact on bone metabolism [12]. In this study, we were able to obtain positive expression of osteogenic marker; Osteopontin. Also, positive Alizarin Red staining was detected after 7 days of culture in the proposed combination of SV and ALN and this is a relatively short duration compared to the 14 days usually taken by the routinely used formulation of osteogenic medium. To the best of our knowledge, no previous studies have elucidated the effect of simultaneous administration of both agents on bone build up. Our team has previously studied the anticarcinogenic effect of this combination on oral squamous cell carcinoma [8]. In this study, we further illustrate an additional impact of this pharmacological combination. Our novel findings shall be meaningful if tested and applied in the context of osteosarcomas or bone metastasis. These aggressive cancers occurring in bone and osteolytic conditions represent a challenge for surgeons, especially when determining the appropriate borders of these tumors. By introducing this combination of SV and ALN, the amount of bone excised from the bony site surrounding the tumor can be minimized. Combining both drugs shall exhibit a dual impact through acting as anti-carcinogenic agent useful in destroying any cancer cells left in the field while inducing osteogenic differentiation of stem cells normally present. In the head-and-neck cancer involving bony structures, the postsurgical use of obturators was part of the treatment regimen which caused additional psychological load for many patients. We present a substitute to these regimens by a combination that may remove hidden cancer cells and, at the same time, help to restore lost bone. To the best of our knowledge, the presented study is the only in vitro study that examined this combination of drugs; further studies are recommended to validate these results and test the efficacy in vivo.

\section{CONCLUSION}

The combined effect of ALN and SV may represent a novel pharmacological approach in treating bone metastasis and, at the same time, treating cancer itself. The synergistic cytotoxic effect previously described by our team should be taken into consideration. In the future, this pharmacological combination may provide a novel treatment modality for bone tissue regeneration applications.

\section{AUTHORS' CONTRIBUTIONS}

Riham M. Aly: Responsible for study design, isolating and propagating stem cells, cell viability testing, and revising the manuscript; Marwa M. Ellithy: Responsible for study design, isolating and propagating stem cells, cell viability testing, and writing the manuscript; and Dina Sabry: Responsible for molecular analysis and revising the manuscript.

\section{CONFLICTS OF INTEREST}

The authors declare that they have no conflicts of interest.

\section{REFERENCES}

1. Vincenzi B, Santini D, Avvisati G, Baldi A, Cesa AL, Tonini G, et al. Statins may potentiate bisphosphonates anticancer properties: A new pharmacological approach? Med Hypotheses 2003;61:98-101.

2. Duque G, Rivas D. Alendronate has an anabolic effect on bone through the differentiation of mesenchymal stem cells. J Bone Miner Res 2007;22:1603-11.

3. Pavan LM, Rêgo DF, Elias ST, De Luca Canto G, Guerra EN. In vitro anti-tumor effects of statins on head and neck squamous cell carcinoma: A Systematic review. PLoS One 2015;10:e0130476. 
4. Daisy P, Suveena SA. Review on comprehensive cancer pathways. Int J Appl Pharm 2014;6:1-5.

5. Pagkalos J, Cha JM, Kang Y, Heliotis M, Tsiridis E, Mantalaris A, et al. Simvastatin induces osteogenic differentiation of murine embryonic stem cells. J Bone Miner Res 2010;25:2470-8.

6. Alexander IM. Pharmacotherapeutic management of osteoprosis and osteopenia. Nurse Pract 2009;34:30-40.

7. Tai IC, Wang YH, Chen CH, Chuang SC, Chang JK, Ho ML, et al. Simvastatin enhances rho/actin/cell rigidity pathway contributing to mesenchymal stem cells' osteogenic differentiation. Int J Nanomedicine 2015;10:5881-94.

8. Abd-el-Fattah DS, Ellithy MM, Aly RM. Synergistic cytotoxic effect of statins and bisphosphonates on squamous cell carcinoma cell line. Int J Pharm Pharm Sci 2017;9:123.
9. Sofi MS, Nabi S. Induction of caspase-3 dependent apoptosis, cell cycle arrest and cytotoxicity in breast cancer cells by abrus precatorius. Int $\mathrm{J}$ Pharm Pharm Sci 2018;10:29-35.

10. Durmus M, Karaaslan E, Ozturk E, Gulec M, Iraz M, Edali N, et al. The effects of single-dose dexamethasone on wound healing in rats. Anesth Analg 2003;97:1377-80.

11. Ellithy M, Ayoub M, Abbas E, El Hamid MA, Baghdadi H, El Fattah DA. Assessment of the osteogenic potential of alendronate on isolated adipose-derived stem cells: An ex-vivo and in-vivo study. J Arab Soc Med Res 2015;10:32-6.

12. Sonobe M, Hattori K, Tomita N, Yoshikawa T, Aoki H, Takakura Y, et al. Stimulatory effects of statins on bone marrow-derived mesenchymal stem cells. Study of a new therapeutic agent for fracture. Biomed Mater Eng 2005;15:261-7. 